\title{
Impaired phagocytosis and reactive oxygen species production in phagocytes is associated with systemic vasculitis
}

Åsa CM Johansson ${ }^{1,2^{*}}$, Sophie Ohlsson ${ }^{3}, \AA$ Åsa Pettersson ${ }^{3}$, Anders A. Bengtsson ${ }^{4}$, Daina Selga ${ }^{3}$, Markus Hansson $^{1}$ and Thomas Hellmark ${ }^{3}$

\begin{abstract}
Background: Anti-neutrophil cytoplasmic antibodies associated vasculitides (AAV) is a group of autoimmune diseases, characterized by small vessel inflammation. Phagocytes such as neutrophils and monocytes are the main effector cells found around the inflamed vessel wall. Therefore, we wanted to investigate aspects of function and activation of these cells in patients with AAV.

Methods: Flow cytometry was used to evaluate: the expression of activation markers (CD11C, CD62L, CD177 and (5aR); the number of recently released neutrophils from bone marrow, defined as CD10'D16 low cells in peripheral blood; and the capacity of peripheral blood monocytes and polymorphonuclear leukocytes (PMN) to produce reactive oxygen species and to phagocytose opsonized bacteria.

Results: AAV patients $(n=104)$ showed an increase of CD10'CD16 low neutrophils and total PMN in peripheral blood, suggesting a combination of increased bone marrow release and prolonged survival. An increased percentage of AAV PMN expressed CD177 but no other signs of activation were seen. A decreased production of reactive oxygen species was observed in AAV phagocytes, which was associated with disease activity. Moreover, granulocytes from patients with microscopic polyangiitis showed lower oxidative burst capacity compared to patients with granulomatosis with polyangiitis or eosinophilic granulomatosis with polyangiitis. In addition, decreased phagocytosis capacity was seen in PMN and monocytes.
\end{abstract}

Conclusion: Our results indicate that phagocytes from AAV patients have impaired function, are easily mobilized from bone marrow but are not particularly activated. The association between low reactive oxygen species formation in PMN and disease severity is consistent with findings in other autoimmune diseases and might be considered as a risk factor.

Keywords: ANCA, Vasculitis, PMN, Monocytes, Oxidative burst, Phagocytosis

\section{Background}

Anti-neutrophil cytoplasmic antibody associated vasculitides (AAV) is a group of autoimmune diseases comprising eosinophilic granulomatosis with polyangiitis (EGPA), granulomatosis with polyangiitis (GPA) and microscopic polyangiitis (MPA). AAV are conditions defined by autoimmune small vessel inflammation [1] and characterized

\footnotetext{
* Correspondence: asa.johansson@med.lu.se

'Department of Haematology, Lund University and Skåne University Hospital, BMC B13, 22184 Lund, Sweden

2University and Regional Laboratories Region Skåne, Clinical Immunology and Transfusion Medicine, Skåne, 22185 Lund, Sweden

Full list of author information is available at the end of the article
}

by autoantibodies, the anti-neutrophil cytoplasmic antibodies (ANCA). The ANCA antigens proteinase 3 (PR3) and myeloperoxidase (MPO) are mainly present in the primary granules of neutrophils and in the peroxidase positive lysosomes of monocytes.

Neutrophils are found in abundance in and around inflamed vessel walls in patients with AAV, and ANCA bound to the target antigens leads to activation of neutrophil effector functions and ultimately tissue damage [2]. In vitro experiments show that ANCA-mediated activation of primed neutrophils leads to degranulation and production of reactive oxygen species (ROS), complement 
activation [3], and release of neutrophil extracellular traps (NETs) [4]. Furthermore, the pathological role of ANCA has been shown in animal models where ANCA cause vasculitis-like symptoms $[5,6]$.

Monocytes have been less studied than neutrophils, but are known to be prominent in AAV lesions [7]. There are reports showing that ANCA can activate monocytes and early exudative macrophages but not mature macrophages [8-10].

Monocytes and PMN, such as neutrophils, are produced in the bone marrow and released into the circulation. Inflammation causes increased mobilization of PMN from the bone marrow that is thought to be reflected by an increased percentage of $\mathrm{CD} 10^{-} \mathrm{CD} 16^{\text {low }}$ neutrophils in peripheral blood $[11,12]$. Upon activation the expression of various surface proteins changes, e.g., C5aR and CD62L are downregulated $[13,14]$ whereas increased CD11c expression is observed [15]. In addition to the changing expression of their surface proteins, activated monocytes and neutrophils are primed to release granules and produce ROS by the nicotinamide adenine dinucleotide phosphate-oxidase (NADPH) complex [16]. ROS are major effector molecules in inflammatory processes and tightly linked to NET formation. During the last decade, an increasing amount of data have been produced to support a $\mathrm{T}$ cell regulating role for monocyte-produced and neutrophil-produced ROS [17-20].
This study aimed to characterize monocytes and PMN from patients with $\mathrm{AAV}$, in regard to function, bone marrow release and activation, in order to understand the role of these phagocytes in AAV and autoimmunity.

\section{Methods}

\section{Patients and controls}

Patients with AAV were recruited to the study when attending their scheduled visit at the Department of Nephrology or Rheumatology, Skåne University Hospital, Lund, Sweden. Classification of AAV was done according to the consensus methodology described by Watts et121 al. in 2007 [21]. Patients with AAV $(\mathrm{n}=104)$ included in the study comprised 73 patients with GPA, median age 66 years (range $20-84$ years), 23 patients with MPA, median age 72 years (range 20-84 years), and 8 patients with EGPA, median age 71 years (range 56-78 years). Patients with EGPA in this study had normal eosinophil counts at the time of sampling, with a mean of $0.2 \times 10^{9} / \mathrm{L}$, range $0.01-0.6 \times 10^{9} / \mathrm{L}$ (reference range $\left.<0.7 \times 10^{9} / \mathrm{L}\right)$. Disease activity was assessed using the Birmingham Vasculitis Activity Score version 3 (BVAS 3) [22]. Demographic and clinical characteristics are shown in Table 1. ANCA specificity was determined with ELISA at an accredited clinical laboratory (Wieslab AB, Malmö, Sweden).

Table 1 Patients characteristics and demographics

\begin{tabular}{|c|c|c|c|}
\hline & All patients $(n=104)$ & Remission BVAS3 = $0(n=82)$ & Disease activity BVAS3 $>1 \quad(n=22)$ \\
\hline Age, years, median (range) & $68(20-86)$ & $68(25-84)$ & $66(20-86)$ \\
\hline Disease duration, years, median (range) & $7(0-50)$ & $7(0-50)$ & $1(0-31)$ \\
\hline GPA, \% (n) & $70 \%(n=73)$ & $68 \%(n=56)$ & $78 \%(n=18)$ \\
\hline $\mathrm{MPA}, \%(n)$ & $22 \%(n=23)$ & $24 \%(n=19)$ & $17 \%(n=4)$ \\
\hline EGPA, \% (n) & $8 \%(n=8)$ & $8 \%(n=7)$ & $4 \%(n=1)$ \\
\hline MPO-ANCA, \% (n) & $30 \%(n=32)$ & $33 \%(n=26)$ & $26 \%(n=6)$ \\
\hline PR3-ANCA, \% (n) & $59 \%(n=61)$ & $56 \%(n=46)$ & $70 \%(n=16)$ \\
\hline BVAS3, median (range) & $0(0-16)$ & 0 & $4(1-16)$ \\
\hline Leukocytes, $10^{9} / \mathrm{L}$, median (range) ${ }^{a}$ & $6.4(3.0-13.7)$ & $6(3.2-13.7)$ & $7.9(3.0-12)$ \\
\hline P-creatinine, $\mu \mathrm{mol} / \mathrm{L}$, median (range) & $98(54-646)$ & $96(59-635)$ & $143(54-646)$ \\
\hline P-CRP, mg/L, median (range) & $2.6(<0.6-92)$ & $2.6(<0.6-27)$ & $5.7(<0.6-92)$ \\
\hline \multicolumn{4}{|l|}{ Treatment ${ }^{\mathrm{b}}$} \\
\hline Prednisone, \% (median dose of treated patients) & $60 \%(6.25 \mathrm{mg})$ & $55 \%(5 \mathrm{mg})$ & 77 \% (15 mg) \\
\hline Azathioprine, \% (n) & $28 \%(n=29)$ & $31 \%(n=26)$ & $13 \%(n=3)$ \\
\hline Mycophenolate mofetil, \% ( $n)$ & $8 \%(n=8)$ & $10 \%(n=8)$ & 0 \\
\hline Rituximab, \% ( $n)$ & $19 \%(n=20)$ & $19 \%(n=17)$ & $17 \%(n=3)$ \\
\hline Methotrexate, \% (n) & $14 \%(n=15)$ & $14 \%(n=11)$ & $17 \%(n=4)$ \\
\hline Cyclophosphamide, \% (n) & $11 \%(n=11)$ & $7 \%(n=6)$ & $27 \%(n=6)$ \\
\hline
\end{tabular}

BVAS Birmingham Vasculitis Activity Score version 3, GPA granulomatosis with polyangiitis, MPA microscopic polyangiitis, EGPA eosinophilic granulomatosis with polyangiitis, MPO myeloperoxidase, ANCA anti-neutrophil cytoplasmic antibodies, $P R 3$ proteinase 3, CRP C-reactive protein. ${ }^{a}$ Reference range $3.5-8.810^{9} / L$. ${ }^{\mathrm{b}}$ There were 14 patients who did not receive any treatment 
As disease controls, patients $(n=26)$ with systemic lupus erythematosus (SLE) were recruited to the study, when attending their scheduled visit at the Department of Rheumatology, Skåne University Hospital, Lund, Sweden. All patients fulfilled at least four American College of Rheumatology classification criteria for SLE [23]. Disease activity was assessed using the Systemic Lupus Erythematosus Disease Activity Index 2000 (SLEDAI-2 K) [24], and organ damage was evaluated according to the Systemic Lupus International Collaborative Clinics/American College of Rheumatology damage index (SLICC/ACR-DI) [25]. Blood donors (Blood center in Lund) and healthy volunteers were recruited as controls $(n=112)$. Demographic data on age and gender were available for 102 of the 112 controls, giving a median age of 49 years (range 19-74) and female to male ratio of 1.1 to 1.0. Patients with AAV and controls were analyzed in parallel between 2011 and 2014. Patients with SLE were analyzed in parallel with healthy controls and Patients with AAV during 2014. The Regional Ethics Board in Lund, Sweden (LU) approved the study and informed consent was obtained from all participants.

\section{Phagocytosis and oxidative burst}

Peripheral blood from patients and controls was collected in vacutainer tubes containing sodium heparin (Becton Dickinson, BD, New York, NY, USA). All samples were analyzed within $24 \mathrm{~h}$. The samples were stored at room temperature and protected from light until analyzed. Phagocytosis was investigated using the in vitro diagnostic (IVD)-labeled PhagoTest assay (Glycotope Biotechnology, $\mathrm{GmBH}$, Germany), according to the manufacturer's protocol. This flow-cytometry-based method measures the percentage of PMN and monocytes having ingested fluorescein-labeled opsonized Escherichia coli (E. coli), and mean fluorescence intensity (MFI) corresponds to the number of ingested bacteria per cell. Production of ROS in peripheral blood PMN and monocytes was investigated using the IVD-labeled PhagoBurst assay, (Glycotope Biotechnology, GmBH, Germany), according to the manufacturer's protocol, after ex vivo activation with phorbol-12-myristate-13-acetate (PMA) or opsonized E. coli. At least 15,000 PMN were collected based on forward and side scatter properties. No patient had ROS deficiency.

\section{Phagocyte phenotypes}

Peripheral blood from patients and controls was collected in vacutainer tubes containing sodium heparin (Becton Dickinson, BD, New York, USA). All sample were analyzed within $24 \mathrm{~h}$. The samples were stored at room temperature and protected from light until analyzed. The expression of selected surface markers on phagocytes was analyzed using flow cytometry. Briefly, peripheral blood was lysed, using $0.84 \%$ ammonium chloride. The remaining leukocytes were stained for surface expression of CD10, CD16, CD62L (BD Bioscience, San Jose, CA, USA), C5aR (CD88), CD11c (Biolegend, San Diego, CA, USA) and CD177 (AbD Serotec, Raleigh, NC, USA) and finally analyzed using a FACSCanto II and the DIVA software (Becton Dickinson, Franklin Lakes, NJ, USA). Gating strategies are shown in Additional file 1: Figure S1.

\section{Statistical analysis}

Correlation was assessed by Spearman's test. The MannWhitney $U$ test was used for two-group comparisons, and the Kruskal-Wallis and Dunn multiple comparisons test was for three or more groups. All $p$ values were considered significant at $p<0.05$. Prism for MacOS version $5.0 \mathrm{a}$ was used for statistic calculations.

\section{Results}

\section{Patient characteristics}

The clinical and demographic characteristics of the patients with AAV $(n=104)$ at the time of sampling are reported in Table 1 . The majority of the patients were diagnosed with GPA (70 \%), whereas patients with MPA and EGPA represented $22 \%$ and $8 \%$ of the cohort, respectively. Most patients were in remission and a little over $20 \%$ of the patients had some kind of disease activity according to the BVAS3, with a median score of 4 (range 1-16). The median age of the patients with SLE was 62 years (range 25-85) and the female-to-male ratio was 3.5 to 1.0. Most SLE patients were in remission or had low disease activity with a mean SLEDAI-2 K of 2 (range 0-6). The disease severity, defined as organ damage, was evaluated using SLICC with a median score of 2 (range 0-6). Of the SLE patients, $60 \%$ were treated with prednisone (the mean dose in treated patients was $5 \mathrm{mg} /$ day, range $2.5-20.0 \mathrm{mg} /$ day); $64 \%$ were treated with hydroxychloroquine and $54 \%$ were treated with other immune modulating drugs (methotrexate, mycophenolate mofetil or azathioprine).

\section{Signs of increased mobilization of PMN from bone marrow in patients with AAV}

The frequency of phagocytes and lymphocytes was evaluated in peripheral blood from patients with AAV $(n=104)$ and healthy controls $(n=112)$. Patients had an increased percentage of PMN and a decreased number of lymphocytes compared with controls $(p<0.0001)$. There was no difference in the number of monocytes (Table 2).

Elevated numbers of $\mathrm{CD} 10^{-} \mathrm{CD} 16^{\text {low }} \mathrm{PMN}$ in peripheral blood are thought to reflect increased mobilization of neutrophils from the bone marrow [11, 12]. Patients with AAV had a greater percentage of CD $10^{-} \mathrm{CD} 16^{\text {low }}$ PMN ( $p=0,0079)$ (Table 2$)$, suggesting elevated bone 
Table 2 Monocyte and PMN phenotypes

\begin{tabular}{|c|c|c|c|}
\hline Phenotype & $\begin{array}{l}\text { Patients } \\
\text { with AAV }\end{array}$ & $\begin{array}{l}\text { Healthy } \\
\text { controls }\end{array}$ & $P$ value \\
\hline Monocytes (\% of leukocytes) & $4.4 \pm 0.3$ & $4.8 \pm 0.24$ & n.s. \\
\hline$\left(10^{9} / L\right.$, reference range $\left.<1,1\right)$ & $0.33 \pm 0.02$ & & \\
\hline Lymphocytes (\% of leukocytes) & $20 \pm 1.2$ & $31 \pm 1.2$ & $<0.0001$ \\
\hline$\left(10^{9} / \mathrm{L}\right.$, reference range $\left.1.1-4.8\right)$ & $0.74 \pm 0.05$ & & \\
\hline PMN (\% of leukocytes) & $39 \pm 1.3$ & $28 \pm 1.1$ & $<0.0001$ \\
\hline$\left(10^{9} / \mathrm{L}\right.$, reference range $\left.1.7-8.0\right)$ & $5.9 \pm 0.3$ & & \\
\hline $\mathrm{CD} 16^{+} \mathrm{CD} 10^{+}(\%$ of $\mathrm{PMN})$ & $84 \pm 1.1$ & $82 \pm 1.2$ & n.s. \\
\hline $\mathrm{CD}_{16}{ }^{\mathrm{dim}} \mathrm{CD} 10^{-}$(\% of PMN) & $8.9 \pm 0.8$ & $6.8 \pm 0.6$ & 0.0109 \\
\hline $\mathrm{CD} 177^{+}(\%$ of $\mathrm{PMN})$ & $55 \pm 2.4$ & $47 \pm 1.9$ & 0.0075 \\
\hline \multicolumn{4}{|l|}{$\mathrm{CD}_{10}{ }^{+} \mathrm{CD} 16^{+} \mathrm{PMN}$} \\
\hline $\mathrm{CD} 88^{+}$(geoMFl) & $442 \pm 14$ & $409 \pm 15$ & n.s \\
\hline $\mathrm{CD} 2 \mathrm{~L}^{+}($geoMFI) & $1,277 \pm 37$ & $1,269 \pm 41$ & n.s. \\
\hline CD11c ${ }^{+}($geoMFl) & $558 \pm 14$ & $596 \pm 16$ & n.s \\
\hline \multicolumn{4}{|l|}{$\mathrm{CD} 10^{+} \mathrm{CD} 16^{\mathrm{dim}} \mathrm{PMN}$} \\
\hline $\mathrm{CD} 88^{+}$(geoMFI) & $391 \pm 8.2$ & $381 \pm 7.6$ & $n . s$ \\
\hline $\mathrm{CD} 2 \mathrm{~L}^{+}($geoMFI) & $450 \pm 23$ & $457 \pm 22$ & n.s \\
\hline 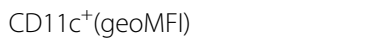 & $354 \pm 7.2$ & $428 \pm 10$ & $<0.0001$ \\
\hline \multicolumn{4}{|l|}{ Monocytes } \\
\hline $\mathrm{CD}_{8} 8^{+}$(geoMFI) & $162 \pm 2.5$ & $165 \pm 2.5$ & n.s \\
\hline $\mathrm{CD} 2 \mathrm{~L}^{+}$(geoMFI) & $770 \pm 29$ & $651 \pm 37$ & $<0.0001$ \\
\hline CD11c ${ }^{+}($geoMFI) & $1,645 \pm 63$ & $2,079 \pm 78$ & $<0.0001$ \\
\hline
\end{tabular}

The frequencies of monocytes, lymphocytes, polymorphonuclear leukocytes (PMN), $\mathrm{CD} 10^{+} \mathrm{CD}_{16}^{+}$(mainly segment nucleated neutrophils) and $\mathrm{CD} 10^{-}$ CD16 ${ }^{\text {dim }}$ (suggested as a marker for newly released neutrophils) were investigated in healthy controls $(n=109)$ and patients $(n=105)$ using flow cytometry. In addition, the level of surface expression of CD88 (C5aR), CD62L and $\mathrm{CD} 11 \mathrm{c}$ was studied on $\mathrm{CD} 10^{+} \mathrm{CD} 16^{+} \mathrm{PMN}, \mathrm{CD} 10^{+} \mathrm{CD} 16^{\mathrm{dim}} \mathrm{PMN}$ and monocyte populations, as a measurement of activation and reported as geometric mean fluorescence intensity (geoMFI). The two-sided Mann-Whitney test was used to calculate the level of significance. Values are reported as mean \pm SEM. AAV anti-neutrophil cytoplasmic antibodies associated vasculitides, n.s. not significant

marrow release. There was no difference in the percentage of mature segment nucleated $\left(\mathrm{CD} 10^{+} \mathrm{CD} 16^{+}\right)$ neutrophils.

In neutrophils, surface translocation of proteinase 3 (PR3), one of the main ANCA targets, appears to occur in association with CD177 [26-28]. In AAV-PMN there were increased levels of CD177 on neutrophils $(p=0.0061)$ (Table 2), which could indicate increased delivery of PR3 to the cell surface of neutrophils. No CD177 was observed on monocytes (data not shown).

To characterize the activation status of phagocytes in peripheral blood, the expression of CD88 (C5aR), CD11c, and $\mathrm{CD} 62 \mathrm{~L}$ were investigated. Mature segment nucleated neutrophils $\left(\mathrm{CD} 10^{+} \mathrm{CD} 16^{+}\right)$from patients with AAV had similar surface expression of these molecules as controls (Table 2). Less surface expression of CD11c was observed on the $\mathrm{CD} 10^{+} \mathrm{CD} 16^{\mathrm{dim}} \mathrm{PMN}$ and monocytes from patients with AAV. AAV monocytes also had increased expression of CD62L (Table 2). Thus, phagocytes in patients with AAV were not more activated than in healthy controls.

\section{Decreased production of ROS in AAV phagocytes}

ROS production is an important effector function of phagocytes for defense against microbes, but also in the regulation of the innate and adaptive immune system $[19,29]$. Production of ROS in phagocytes is generated by the NADPH oxidase that resides in the plasma membrane (5\%) and in granule membranes (95\%) in neutrophils. To evaluate phagocyte function in AAV, we decided to investigate intracellular ROS production. PMN and monocytes in peripheral whole blood from patients with AAV $(n=104)$ (Table 1$)$, SLE $(n=26)$, and healthy controls $(n=112)$, were stimulated with either the protein kinase $\mathrm{C}$ activator, PMA, or with opsonized E. coli. AAV phagocytes, both PMN and monocytes, showed decreased capacity to produce ROS after activation with phorbol 12 -myristate 13 -acetate (PMA) $(p<0.0001)$ or $E$. coli $(p<0.0001$, Fig. 1a and b) compared with healthy controls.

PMN from patients with AAV had less ROS formation compared with PMN from patients with SLE after E. coli stimulation $(p<0.0001)$ (Fig. 1a), but no significant difference was observed after PMA activation.

The patients were divided based on disease characteristics into EGPA, GPA, or MPA. PMN from patients with MPA ( $\mathrm{n}=23$ ) had decreased burst capacity compared to GPA $(\mathrm{n}=73 ; p<0.05)$ and EGPA patients $(\mathrm{n}=8 ; p<0.05)$ after PMA activation (Fig. 2), whereas no significant differences were observed after $E$. coli stimulation (data not shown). The ROS producing capacity was not associated with the presence of PR3-ANCA or MPO-ANCA (data not shown).

\section{Decreased ROS formation is associated with disease activity}

The severity of autoimmune diseases has previously been associated with decreased ROS production [30-32]. Hence, to study if the disease activity of AAV was associated with changes in ROS production, the patients were divided in two groups based on disease activity according to the BVAS3 (Table 1). PMN from patients with BVAS3 $\geq 1$ had decreased ROS production, compared with patients in remission, when activated with $E$. coli $(p=0,0306)$ (Fig. 3a) and PMA $(p=0,0471)$. Most patients in the disease activity group were diagnosed as having GPA $(n=17)$ (Table 1$)$. There was no association between disease activity and ROS production in monocytes (data not shown).

Prednisone treatment is common in this patient group and affects phagocyte function. In this study, $60 \%$ of the patients were on prednisone treatment, with a mean dose of $7.5 \mathrm{mg}$ (range $2-80 \mathrm{mg}$ ) (Table 1). To investigate the corticosteroid effect on ROS production, 


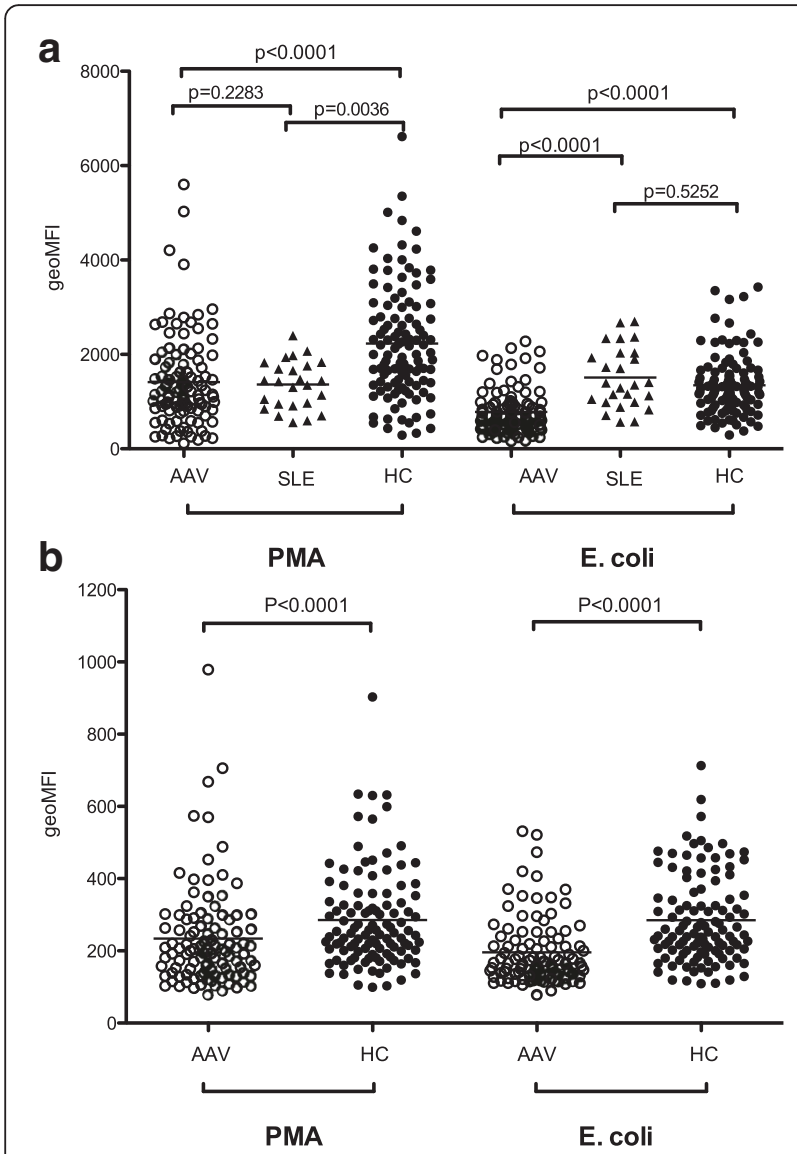

Fig. 1 Phagocytes from patients with anti-neutrophil cytoplasmic antibodies associated vasculitides (AAV) produced fewer reactive oxygen species (ROS) than phagocytes from healthy blood donors. The capacities of polymorphonuclear leukocytes (PMN) (a) or monocytes (b) from healthy controls $(H C)(n=112)$, patients with AAV $(n=104)$, and patients with systemic lupus erythematosus (SLE) $(n=26)$ (only PMN) to produce ROS upon activation with phorbol 12-myristate 13-acetate (PMA) or opsonized E. coli were investigated using flow cytometry. The amount of ROS produced is shown as geometric mean fluorescence intensity (geoMF). The two-sided Mann-Whitney test was used to calculate the level of significance. Horizontal lines represent the median value of each dataset

the patients with disease activity (BVAS3 $\geq 1$ ) or in remission (BVAS3 $=0$ ) were divided based on prednisone treatment or not (Fig. 3b). Patients in remission without prednisone had a higher burst capacity compared with patients on prednisone treatment after $E$. coli stimulation $(p<0.01)$. However, patients in remission who were not receiving prednisone treatment still had a decreased burst compared to healthy controls $(p<0.05)$. In relation to corticosteroid treatment, there was no difference in burst capacity in patients with BVAS $\geq 1$. Similar results were seen after PMA activation. One patient in the group with disease activity was newly diagnosed and not on any treatment at the time of sampling. PMN from this patient had decreased burst

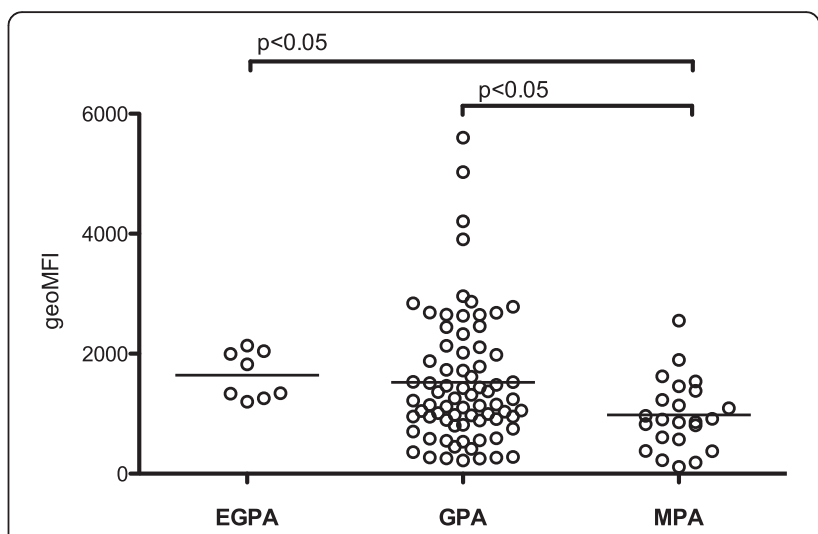

Fig. 2 Patients with microscopic polyangiitis (MPA) had lower reactive oxygen species (ROS) formation compared with patients with granulomatosis with polyangiitis (GPA) or eosinophilic granulomatosis with polyangiitis (EGPA). ROS production was measured by flow cytometry after ex vivo activation of peripheral blood PMN with phorbol 12-myristate 13-acetate. Patients with antineutrophil cytoplasmic antibodies associated vasculitides were divided based on disease characteristics into EGPA, GPA or MPA. The amount of ROS produced is shown as geometric mean fluorescence intensity (geoMF). The Kruskal-Wallis test with Dunn's multiple comparison test was used to calculate the level of significance. Line represents the median value of each dataset

activity (geometric mean fluorescence intensity of 875 and 1053 after E. coli or PMA activation, respectively). In line with the PMN, the monocytes from this patient also produced decreased amounts of ROS (171 and 218 after $E$. coli or PMA activation, respectively (Fig. 3b).

\section{Decreased phagocytosis in patients with AAV}

Another important function of phagocytes is the phagocytosis of antibody-coated microbes and foreign material, which often precedes the intracellular ROS production. The capacity to phagocytose opsonized E. coli was investigated in PMN and monocytes from patients with AAV $(n=83)$ or SLE $(n=26)$ and controls $(n=54)$. Both AAV PMN and monocytes had decreased phagocytosis capacity, defined as the amount of phagocytosed E. coli per cell compared with patients with SLE and healthy controls $(p<0.0001)$ (Fig. 4a). The AAV PMN also had a decreased percentage of phagocytosing cells $(p<0.0001)$ (Fig. 4b). There was no association with disease activity, corticosteroids or ANCA specificity (data not shown).

\section{Discussion}

PMN and monocytes from patients with AAV were characterized with respect to bone marrow release, activation and function. This study shows that patients with AAV have increased mobilisation of neutrophils from the bone marrow, impaired phagocyte functions, such as ROS production and phagocytosis, but normal levels of most phagocyte activation markers. 


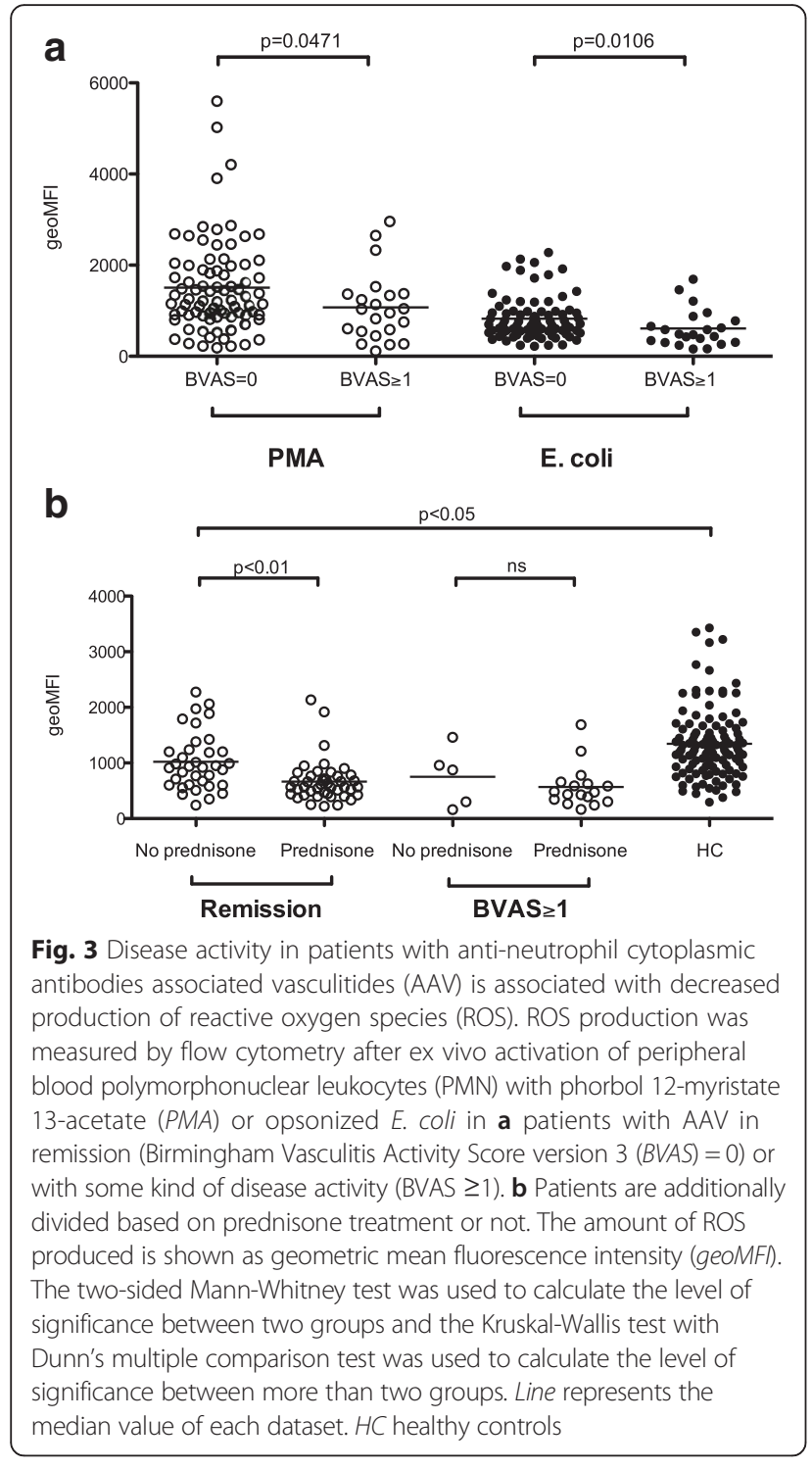

In line with earlier observations, we found that patients with AAV had a decreased percentage of lymphocytes and an increase of PMN. Low levels of lymphocytes are common on diagnosis of AAV and during flares [33] and might also be an effect of immune suppressive treatment $[34,35]$. The increased percentage of PMN in patients with AAV could be explained by increased survival in the periphery in combination with elevated bone marrow release. We have shown previously that AAV neutrophils have a lower rate of apoptosis and longer in vitro survival compared with healthy controls and patients with SLE [36]. Furthermore, patients with AAV appeared to have an increased release of neutrophils from the bone marrow, measured as elevated numbers of $\mathrm{CD} 16^{\mathrm{dim}} \mathrm{CD} 10^{-}$neutrophils in peripheral blood. Increased levels of CD177 were observed on PMN, but there were no other obvious signs

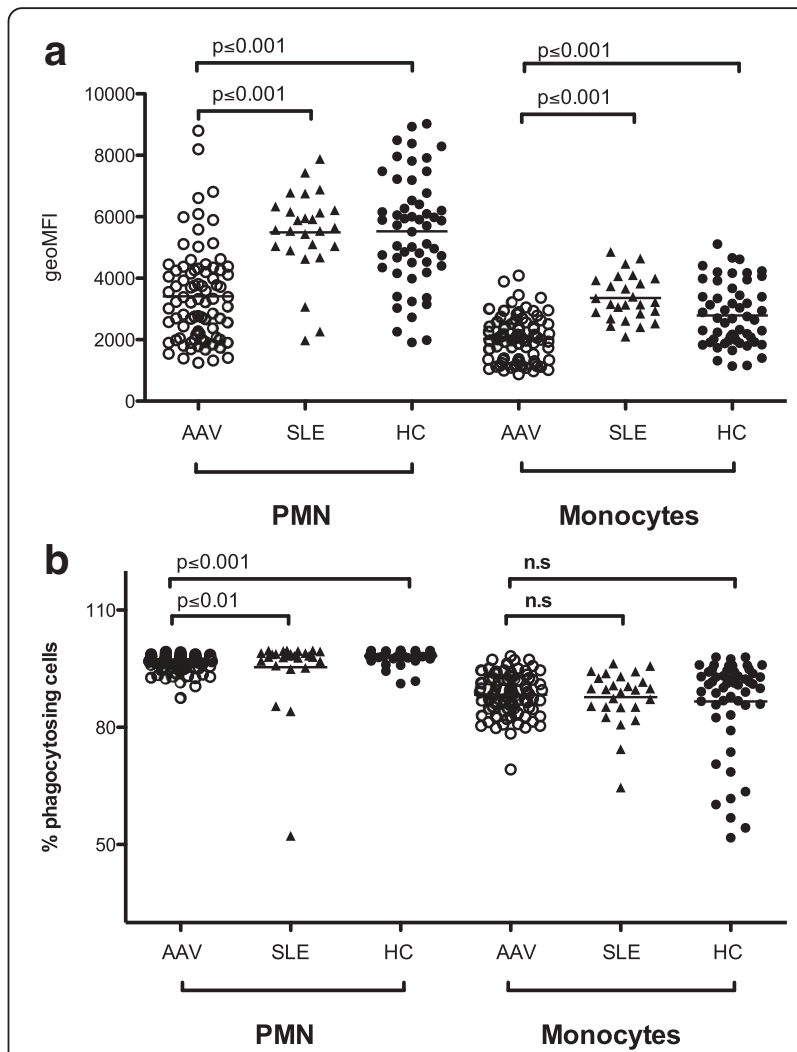

Fig. 4 Decreased phagocytosis in anti-neutrophil cytoplasmic antibodies associated vasculitides (AAV). To evaluate further the function of phagocytes in AAV, the capacity to phagocytose opsonized E. coli was investigated in polymorphonuclear leukocytes (PMN) and monocytes from patients with AAV $(n=84)$, patients with systemic lupus erythematosus $(n=26)$, and healthy controls $(H C)(n=54)$. a Amount of phagocytosed E. coli bacteria shown as geometric mean fluorescence intensity (geoMFI). b Percentage of phagocytosing cells. Differences between healthy controls, patients with SLE, and patients with AAV were calculated using the Kruskal-Wallis test with Dunn's multiple comparison test, and the following $p$ values were obtained for all three groups: geoMFI of PMN, $p<0.0001$; geoMFI of monocytes, $p<0.0001$ and \% phagocytosing cells of PMN, $p<0.0001$; and \% phagocytosing cells of monocytes, $p=0.4091$.

$P$ values presented in the figure are for comparison between two groups

of activation. The elevated expression of CD177 on PMN is in concordance with prior findings $[26,27]$.

Our data support that patients with AAV have a decreased capacity to produce ROS in vivo. The association with disease activity further strengthened our finding. Low ROS production has been associated with disease severity in other autoimmune conditions, including Behcet's disease [30], Guillian-Barre syndrome [31], multiple sclerosis [32] and SLE [37], and might be a common denominator in maintaining chronic inflammation. Moreover, recent findings in an ANCA-induced glomerulonephritis model using mice deficient in either of the NADPH oxidase subunits, gp $91^{\text {phox }}$ or $\mathrm{p} 47^{\text {phox }}[38]$, suggested that ROS limit ANCA-induced inflammation by downregulating 
caspase 1 and hence keep the inflammasome in check. This finding supports an important role of ROS in regulating vasculitis.

In patients with AAV, monocytes and PMN had decreased capacity to phagocytose compared to patients with SLE. This effect could not be explained by differences in age or treatment between the patient cohorts. Furthermore, patients with AAV tended towards more compromised burst formation than patients with SLE. Using, the same methods as in this report, Taylor et al. saw a similar scenario in which patients with subacute liver failure had decreased phagocytosis, whereas patients with acute liver failure or sepsis did not differ from healthy controls [39]. Future studies are needed to explain these differences.

Patients with MPA had decreased ROS production compared to patients with GPA or EGPA, suggesting more impaired phagocyte function in these patients. The patients with MPA were slightly older than those with GPA (median age 72 and 66 years, respectively) and an age-dependent difference in the effect on ROS production in these two groups could not be excluded; however, no correlation between age and ROS production was seen (data not shown). Future studies designed to investigate this difference would be of interest.

Observations from a genome-wide association study have suggested that PR3-AAV and MPO-AAV are distinct autoimmune syndromes with different genetic phenotypes [40]. Interestingly, by dividing patients based on ANCA specificity, rather than clinical diagnosis, no differences in ROS production were observed. This indicates that decreased ROS formation might be associated with chronic inflammatory processes giving rise to the clinical picture, rather than specifically linked to ANCA pathogenesis.

Corticosteroids have been reported to affect ROS production in PMN in a cumulative dose-dependent way [41], and it is presently unclear whether this effect is due to increased disease severity. In a previous report on PMN function in SLE there was no correlation between corticosteroid dose and the amount of intracellular ROS produced. However, the patients were receiving relatively low doses of corticosteroids (mean $=5 \mathrm{mg}$ oral prednisone/day in treated patients) [37]. In the present study we observed that patients in remission who were receiving ongoing prednisone treatment had decreased burst compared with patients not on prednisone, suggesting that in this case a minor effect of prednisone on ROS formation could not be excluded. On the other hand, patients with a tendency to recurrent flares or having low grade activity (not captured by the BVAS) frequently remain on prednisone treatment during remissions, hence patients in remission who are on prednisone treatment might have another kind of inflammatory phenotype that is reflected by decreased ROS formation.
In general, prednisone treatment could not explain the decreased ROS production in patients with AAV, as the patients in remission who were not being treated with prednisone had a decreased burst compared with controls. In addition, a newly diagnosed patient who was not on any treatment at the time of sampling had low burst activity.

We have previously reported that PR3-ANCA induced increased intracellular but not extracellular ROS production in PMN from patients with AAV [42]. The activation and control of the NADPH oxidase in neutrophils (NOX2) is incompletely understood. Different agonists encountered by the neutrophil engage different combinations of kinases, and thereby affect the degree of activity of the NADPH complex, and in the end the amount of ROS produced [43]. Hence, the different results in PR3-ANCA-induced ROS on one hand and E. coli or PMA on the other hand, might be due to the different routes of NADPH activation: PR3-ANCA activates by binding to membrane-bound PR3; opsonized E. Coli by receptor mediated phagocytosis; and PMA by activation of protein kinase $\mathrm{C}$.

\section{Conclusions}

The phagocytes in AAV were found to have decreased functions such as lower ROS formation and phagocytosis, compared to healthy controls. The decreased ROS production is consistent with findings in other autoimmune diseases and might be a risk factor in autoimmunity and a common denominator maintaining chronic inflammation. Although increased expression of CD177 was observed on PMN, no other clear signs of activation were seen. The increased percentage of PMN in peripheral blood might be due to a combination of increased bone marrow release and prolonged survival. Future studies will illuminate the role of ROS formation and PMN in AAV and autoimmunity.

\section{Additional file}

Additional file 1: Gating strategies. (DOCX $188 \mathrm{~kb}$ )

\section{Abbreviations}

AAV: anti-neutrophil cytoplasmic antibodies associated vasculitides; ANCA: the anti-neutrophil cytoplasmic antibodies; BVAS3: Birmingham Vasculitis Activity Score version 3; E. coli: Escherichia coli; EGPA: eosinophilic granulomatosis with polyangiitis; ELISA: enzyme-linked immunosorbent assay; GeoMFI: geometric mean fluorescence intensity; GPA: granulomatosis with polyangiitis; IVD: in vitro diagnostic; MPA: microscopic polyangiitis; MPO: myeloperoxidase; NETs: neutrophil extracellular traps; NAPDH: nicotinamide adenine dinucleotide phosphate-oxidase;

PMA: phorbol 12-myristate 13-acetate; PMN: polymorphonuclear leukocytes; PR3: proteinase 3; ROS: reactive oxygen species; SLE: systemic lupus erythematosus; SLEDAI-2 K: Systemic Lupus Erythematosus Disease Activity Index 2000; SLICC/ACR-DI: Systemic Lupus International Collaborative Clinics/ American College of Rheumatology damage index. 


\section{Competing interests}

The authors declare that they have no competing interests.

\section{Authors' contributions}

$\AA \mathrm{P}$ and $\AA \mathrm{CMJ}$ did the laboratory work. $\AA \mathrm{CMJ}$ and TH analyzed the data. SO, $A A B$ and DS gathered all clinical data. $\mathrm{TH}, \mathrm{MH}, \mathrm{SO}, \AA \mathrm{P} \mathrm{P}, \mathrm{AAB}, \mathrm{DS}$, and $\AA \mathrm{CMJ}$ contributed to the design of the study and wrote the manuscript. All authors read and approved the final manuscript.

\section{Acknowledgements and affiliations}

This work was supported by grants from; Alfred Österlund's Foundation, The Crafoord Foundation, Greta and Johan Kock's Foundation, King Gustaf V's 80th Birthday Foundation, Lund University Hospital, the Swedish Rheumatism Association, the Swedish Research council (X65X-15152), and the Foundation of the National Board of Health and Welfare.

\section{Author details}

Department of Haematology, Lund University and Skåne University Hospital, BMC B13, 22184 Lund, Sweden. 'University and Regional Laboratories Region Skåne, Clinical Immunology and Transfusion Medicine, Skåne, 22185 Lund, Sweden. ${ }^{3}$ Department of Clinical Sciences Lund, Nephrology, Lund University, Skane University Hospital, Lund, Sweden. ${ }^{4}$ Department of Clinical Sciences, Lund, Rheumatology, Lund University, Skåne University Hospital, Lund, Sweden.

\section{Received: 27 November 2015 Accepted: 11 April 2016}

\section{Published online: 22 April 2016}

\section{References}

1. Cartin-Ceba R, Peikert T, Specks U. Pathogenesis of ANCA-associated vasculitis. Curr Rheumatol Rep. 2012;14(6):481-93.

2. Kettritz R. How anti-neutrophil cytoplasmic autoantibodies activate neutrophils. Clin Exp Immunol. 2012;169(3):220-8.

3. Xiao H, Schreiber A, Heeringa P, Falk RJ, Jennette JC. Alternative complement pathway in the pathogenesis of disease mediated by anti-neutrophi cytoplasmic autoantibodies. Am J Pathol. 2007;170(1):52-64.

4. Kessenbrock K, Krumbholz M, Schonermarck U, Back W, Gross WL, Werb Z, et al. Netting neutrophils in autoimmune small-vessel vasculitis. Nat Med. 2009;15(6):623-5.

5. Little MA, Smyth CL, Yadav R, Ambrose L, Cook HT, Nourshargh S, et al. Antineutrophil cytoplasm antibodies directed against myeloperoxidase augment leukocyte-microvascular interactions in vivo. Blood. 2005;106(6):2050-8.

6. Xiao H, Heeringa P, Hu P, Liu Z, Zhao M, Aratani Y, et al. Antineutrophil cytoplasmic autoantibodies specific for myeloperoxidase cause glomerulonephritis and vasculitis in mice. J Clin Invest. 2002;110(7):955-63.

7. Jennette JC, Falk RJ, Hu P, Xiao H. Pathogenesis of antineutrophil cytoplasmic autoantibody-associated small-vessel vasculitis. Annu Rev Pathol. 2013;8:139-60.

8. Nowack R, Schwalbe K, Flores-Suarez LF, Yard B, van der Woude FJ. Upregulation of CD14 and CD18 on monocytes In vitro by antineutrophil cytoplasmic autoantibodies. J Am Soc Nephrol. 2000;11(9):1639-46.

9. Weidner S, Neupert W, Goppelt-Struebe M, Rupprecht HD. Antineutrophil cytoplasmic antibodies induce human monocytes to produce oxygen radicals in vitro. Arthritis Rheum. 2001:44(7):1698-706.

10. Wikman A, Fagergren A, Forslid J, Jacobson SH, Johansson SG, Lundahl J. Antineutrophil cytoplasmic antibodies induce decreased CD62L expression and enhanced metabolic activity in monocytes. Scand J Immunol. 2003:57(2):179-84.

11. Kaneko T, Stearns-Kurosawa DJ, Taylor Jr F, Twigg M, Osaki K, Kinasewitz GT, et al. Reduced neutrophil CD10 expression in nonhuman primates and humans after in vivo challenge with E. coli or lipopolysaccharide. Shock. 2003;20(2):130-7.

12. Orr Y, Taylor JM, Bannon PG, Geczy C, Kritharides L. Circulating CD10-/ CD16low neutrophils provide a quantitative index of active bone marrow neutrophil release. Br J Haematol. 2005;131(4):508-19.

13. Furebring $M$, Hakansson L, Venge $P$, Sjolin J. Differential expression of the C5a receptor and complement receptors 1 and 3 after LPS stimulation of neutrophils and monocytes. Scand J Immunol. 2004;60(5):494-9.

14. Smith CW. Leukocyte-endothelial cell interactions. Semin Hematol. 1993;30(4 Suppl 4):45-53. discussion 54-45.
15. Stacker SA, Springer TA. Leukocyte integrin P150,95 (CD11c/CD18) functions as an adhesion molecule binding to a counter-receptor on stimulated endothelium. J Immunol. 1991;146(2):648-55.

16. Bedard K, Krause KH. The NOX family of ROS-generating NADPH oxidases: physiology and pathophysiology. Physiol Rev. 2007;87(1):245-313.

17. Hansson M, Hermodsson S, Brune M, Mellqvist UH, Naredi P, Betten A, et al. Histamine protects T cells and natural killer cells against oxidative stress. J Interferon Cytokine Res. 1999;19(10):1135-44.

18. Hansson M, Romero A, Thoren F, Hermodsson S, Hellstrand K. Activation of cytotoxic lymphocytes by interferon-alpha: role of oxygen radical-producing mononuclear phagocytes. J Leukoc Biol. 2004;76(6):1207-13.

19. Holmdahl R, Sareila O, Pizzolla A, Winter S, Hagert C, Jaakkola N, et al. Hydrogen peroxide as an immunological transmitter regulating autoreactive T cells. Antioxid Redox Signal. 2013;18(12):1463-74.

20. Mellqvist UH, Hansson M, Brune M, Dahlgren C, Hermodsson S, Hellstrand K Natural killer cell dysfunction and apoptosis induced by chronic myelogenous leukemia cells: role of reactive oxygen species and regulation by histamine. Blood. 2000;96(5):1961-8.

21. Watts R, Lane S, Hanslik T, Hauser T, Hellmich B, Koldingsnes W, et al. Development and validation of a consensus methodology for the classification of the ANCA-associated vasculitides and polyarteritis nodosa for epidemiological studies. Ann Rheum Dis. 2007;66(2):222-7.

22. Mukhtyar C, Lee R, Brown D, Carruthers D, Dasgupta B, Dubey S, et al. Modification and validation of the Birmingham Vasculitis Activity Score (version 3). Ann Rheum Dis. 2009;68(12):1827-32

23. Tan EM, Cohen AS, Fries JF, Masi AT, McShane DJ, Rothfield NF, et al. The 1982 revised criteria for the classification of systemic lupus erythematosus. Arthritis Rheum. 1982;25(11):1271-7.

24. Gladman DD, Ibanez D, Urowitz MB. Systemic lupus erythematosus disease activity index 2000. J Rheumatol. 2002;29(2):288-91.

25. Gladman D, Ginzler E, Goldsmith C, Fortin P, Liang M, Urowitz M, et al. The development and initial validation of the Systemic Lupus International Collaborating Clinics/American College of Rheumatology damage index for systemic lupus erythematosus. Arthritis Rheum. 1996:39(3):363-9.

26. Abdgawad M, Gunnarsson L, Bengtsson AA, Geborek P, Nilsson L, Segelmark $M$, et al. Elevated neutrophil membrane expression of proteinase 3 is dependent upon CD177 expression. Clin Exp Immunol. 2010;161(1):89-97.

27. Bauer S, Abdgawad M, Gunnarsson L, Segelmark M, Tapper H, Hellmark T. Proteinase 3 and CD177 are expressed on the plasma membrane of the same subset of neutrophils. J Leukoc Biol. 2007;81(2):458-64.

28. von Vietinghoff S, Eulenberg C, Wellner M, Luft FC, Kettritz R. Neutrophil surface presentation of the anti-neutrophil cytoplasmic antibody-antigen proteinase 3 depends on $\mathrm{N}$-terminal processing. Clin Exp Immunol. 2008:152(3):508-16.

29. Hansson M, Asea A, Ersson U, Hermodsson S, Hellstrand K. Induction of apoptosis in NK cells by monocyte-derived reactive oxygen metabolites. J Immunol. 1996;156(1):42-7.

30. Eksioglu-Demiralp E, Direskeneli $H$, Kibaroglu A, Yavuz S, Ergun $T$, Akoglu T. Neutrophil activation in Behcet's disease. Clin Exp Rheumatol. 2001:19(5 Suppl 24):S19-24.

31. Mossberg N, Andersen O, Nilsson S, Dahlgren C, Hellstrand K, Lindh M, et al. Oxygen radical production and severity of the Guillain-Barre syndrome. J Neuroimmunol. 2007;192(1-2):186-91.

32. Mossberg N, Movitz C, Hellstrand K, Bergstrom T, Nilsson S, Andersen O. Oxygen radical production in leukocytes and disease severity in multiple sclerosis. J Neuroimmunol. 2009:213(1-2):131-4.

33. Izzedine $H$, Cacoub P, Launay-Vacher V, Bagnis C, Deray G. Lymphopenia in Wegener's granulomatosis. A new clinical activity index? Nephron. 2002;92(2):466-71.

34. Fauci AS, Dale DC, Wolff SM. Cyclophosphamide and lymphocyte subpopulations in Wegener's granulomatosis. Arthritis Rheum. 1974;17(4):355-61

35. Zhu LP, Cupps TR, Whalen G, Fauci AS. Selective effects of cyclophosphamide therapy on activation, proliferation, and differentiation of human B cells. J Clin Invest. 1987;79(4):1082-90.

36. Abdgawad M, Pettersson A, Gunnarsson L, Bengtsson AA, Geborek P, Nilsson $L$, et al. Decreased neutrophil apoptosis in quiescent ANCAassociated systemic vasculitis. PLoS One. 2012;7(3):e32439.

37. Bengtsson AA, Pettersson A, Wichert S, Gullstrand B, Hansson M, Hellmark T, et al. Low production of reactive oxygen species in granulocytes is 
associated with organ damage in systemic lupus erythematosus. Arthritis Res Ther. 2014;16(3):R120.

38. Schreiber A, Luft FC, Kettritz R. Phagocyte NADPH oxidase restrains the inflammasome in ANCA-induced GN. J Am Soc Nephrol. 2015;26(2):411-24.

39. Taylor NJ, Nishtala A, Manakkat Vijay GK, Abeles RD, Auzinger G, Bernal W, et al. Circulating neutrophil dysfunction in acute liver failure. Hepatology. 2013; 57(3):1142-52.

40. Lyons PA, Rayner TF, Trivedi S, Holle JU, Watts RA, Jayne DR, et al. Genetically distinct subsets within ANCA-associated vasculitis. N Engl J Med. 2012;367(3):214-23.

41. Fukushima K, Ando M, Ito K, Suga M, Araki S. Stimulus- and cumulative dose-dependent inhibition of O2- production by polymorphonuclear leukocytes of patients receiving corticosteroids. J Clin Lab Immunol. 1990;33(3):117-23.

42. Ohlsson SM, Ohlsson S, Soderberg D, Gunnarsson L, Pettersson A Segelmark M, et al. Neutrophils from vasculitis patients exhibit an increased propensity for activation by anti-neutrophil cytoplasmic antibodies. Clin Exp Immunol. 2014;176(3):363-72

43. El-Benna J, Dang PM, Gougerot-Pocidalo MA. Priming of the neutrophil NADPH oxidase activation: role of p47phox phosphorylation and NOX2 mobilization to the plasma membrane. Semin Immunopathol. 2008;30(3):279-89.

Submit your next manuscript to BioMed Central and we will help you at every step:

- We accept pre-submission inquiries

- Our selector tool helps you to find the most relevant journal

- We provide round the clock customer support

- Convenient online submission

- Thorough peer review

- Inclusion in PubMed and all major indexing services

- Maximum visibility for your research

Submit your manuscript at www.biomedcentral.com/submit
Biomed Central 